The administrative problems of merit and demerit rating are reviewed as are incentives to improve driver education and the use of mechanical safety devices.

On the question of insuring the driver rather than the automobile, the extensive legal and rating difficulties are thought to outweigh any possible advantages.

The overall test for the soundness of a classification rating system is that the rates be reasonable, adequate and not unfairly discriminatory. Classification refinement must stand the test of universal use and public acceptance.

Graduation of Excess Ratio Distributions by the Method of Moments by LEWIS H. ROBERTS.

Retrospective rating, in which premiums are based partly on limited actual losses, depends upon a table showing charges for the expected values of losses in excess of specified loss ratios.

Since the loss ratios of small risks are subject to much wider fluctuation than those of large risks, a table of such charges is a function of both the loss ratio and the size of risk, a measure of size being total expected losses. The wide range of sizes of risk prevents accumulation of sufficient experience to determine the proper charge for any particular size and specified loss ratio without an efficient procedure for graduating the indications of available experience for risks of various recorded sizes.

The method of moments provides a means of effecting such graduation with a minimum number of statistical constants (parameters) to be determined from the experience. In this paper formulas are given for calculating the first four moments for the probability distribution of loss ratios for risks of particular sizes. Additional formulae provide a method of graduating the moments to cover all sizes of risk. Lastly, a procedure is given for converting the graduated moments into a table of charges.

Revision of Rates Applicable to a Class of Property Five Insurance by C. Otis Shaver.

The statistical records maintained in respect to fire insurance in the United States are considerably more extensive than those maintained in other countries and this paper provides a valuable detailed step by step description of the method by which these data are used to revise fire insurance rates.

Starting from premium and loss data developed by occupancy class, construction-protection classification and term of policy, earned premiums and incurred losses are developed for each of the five most recent calandar years. These earned premiums are then adjusted to current rate levels. Using appropriate allowances for expenses and profits, an indicated rate change is developed for each classification and the actual application of the revision explained.

\title{
SOCIETY OF ACTUARIES
}

The 1957 Volume of the Transactions of the Society of Actuaries includes a paper, "Reserves for Individual Hospital and Surgical Expense Insurance" 